\title{
A mixed treatment comparison meta-analysis of metaphylaxis treatments for bovine respiratory disease in beef cattle ${ }^{1,2}$
}

\author{
K. M. Abell,* M. E. Theurer, $\uparrow$ R. L. Larson, $\uparrow \dagger^{3}$ B. J. White, $\uparrow+$ and M. Apley $\$$ \\ *Department of Diagnostic Medicine/Pathobiology, College of Veterinary Medicine, Kansas State \\ University, Manhattan 66506; †Beef Cattle Institute, Kansas State University, Manhattan 66506; \\ and $\ddagger$ Department of Clinical Sciences, College of Veterinary Medicine, Kansas State University, Manhattan 66506
}

\begin{abstract}
The objective of this project was to evaluate the effects of antimicrobials approved for parenteral metaphylactic use in feeder and stocker calves on morbidity and mortality for bovine respiratory disease with the use of a mixed treatment comparison meta-analysis. An initial literature review was conducted in April 2016 through Pubmed, Agricola, and CAB (Commonwealth Agricultural Bureau) for randomized controlled trials for metaphylaxis antimicrobial administered parentally to incoming feedlot or stocker calves within $48 \mathrm{~h}$ of arrival. The final list of publications included 29 studies, with a total of 37 trials. There were 8 different metaphylactic antimicrobials. Final event outcomes were categorized into bovine respiratory disease (BRD) morbidity cumulative incidence $\mathrm{d} 1$ to $\leq$ 60 of the feeding period, BRD morbidity cumulative incidence $\mathrm{d} 1$ to closeout of the feeding period, BRD
\end{abstract}

mortality cumulative incidence $\mathrm{d} 1$ to closeout of the feeding period, and BRD retreatment cumulative incidence morbidity $\mathrm{d} 1$ to closeout of the feeding period. Network meta-analysis combined direct and indirect evidence for all the event outcomes to determine mean odds ratio (OR) with $95 \%$ credibility intervals (CrIs) for all metaphylactic antimicrobial comparisons. The "upper tier" treatment arms for morbidity d 1 to $\leq 60$ included tulathromycin, gamithromycin, and tilmicosin. For BRD mortality cumulative incidence d 1 to closeout and BRD retreatment morbidity d 1 to closeout, classifying the treatment arms into tiers was not possible due to overlapping $95 \%$ CrIs. The results of this project accurately identified differences between metaphylactic antimicrobials, and metaphylactic antimicrobial options appear to offer different outcomes on BRD morbidity and mortality odds in feedlot cattle.

Key words: antimicrobial, bovine respiratory disease, meta-analysis, metaphylaxis, mixed treatment comparison

(C) 2017 American Society of Animal Science. All rights reserved.

J. Anim. Sci. 2017.95:626-635 doi:10.2527/jas2016.1062

\footnotetext{
${ }^{1}$ There was no extra-institutional funding or support for this project. The authors have performed consulting or sponsored research for the following companies: Bayer Animal Health, Boehringer-Ingelheim Vetmedica, CEVA Biomune, Elanco Animal Health, Immvac, Merck Animal Health, Merial Animal Health, MSD, National Cattlemen's Beef Association, Norbrook, Novartis Animal Health, Parnell, Professional Beef Services, USDA, and Zoetis Animal Health. No pharmaceutical companies were involved in design, research, analysis, or manuscript preparation nor provided financial support.

${ }^{2}$ This project was supported by Agriculture and Food Research Initiative Competitive Grant no. 2015-67015-23079 from the USDA National Institute of Food and Agriculture.

${ }^{3}$ Corresponding author: rlarson@vet.k-state.edu

Received September 23, 2016.

Accepted December 19, 2016.
}

\section{INTRODUCTION}

Bovine respiratory disease (BRD) complex is a well-documented, multi-faceted disease syndrome involving environmental factors, host factors, and management practices affecting the health and performance of feedlot calves (Kelly and Janzen, 1986; Smith, 1998). Marketing and shipment of cattle are associated with stress prior to feedlot arrival, which increases the risk for BRD of fed cattle (Lofgreen et al., 1978; Camp et al., 1981). Mass medication, also known as metaphylaxis, has been used to prevent BRD in groups of cattle arriving at feedlots with over half of United States feedlots using metaphylaxis on at least some groups of cattle near the time of feedlot arrival (USDA, 2011). 
Multiple antimicrobials are currently available and used metaphylactically to decrease negative effects of BRD in groups of feedlot cattle, and the decision to implement a specific antimicrobial is dependent on the efficacy and cost effectiveness (Nickell and White, 2010). Clinical trials have been conducted to investigate the efficacy of antimicrobials for the treatment and control of $\mathrm{BRD}$, and metaphylaxis uses have been investigated as a method to reduce morbidity and mortality associated with BRD in feedlot cattle (DeDonder and Apley, 2015; Ives and Richeson, 2015). Meta-analysis and systematic reviews of the available literature have been previously performed to summarize published clinical trials for antimicrobial treatment of clinical BRD cases and for specific antimicrobials used metaphylactically, but no systematic review or meta-analysis has been published that summarizes clinical trials for all approved parenterally administered metaphylactic antimicrobials (Van Donkersgoed, 1992; Wellman and O'Connor, 2007; Wileman et al., 2009; Nickell and White, 2010).

A mixed treatment comparison (MTC) metaanalysis can assess indirect comparisons between antimicrobials where an actual clinical trial was not performed (Higgins and Whitehead, 1996; Lu and Ades, 2004; Jansen et al., 2011). The indirect comparison have been proven to be realistic estimates of disease risk when direct estimates are not available (O'Connor et al., 2016). The MTC of meta-analysis has been done frequently in the human medical field (Mills et al., 2009; Roever and Biondi-Zoccai, 2016; Shao et al., 2016), and has previously evaluated antimicrobial efficacy for treatment of BRD (O'Connor et al., 2013). The objective of this research was to evaluate the effect of parenterally administered metaphylactic antimicrobials approved for feeder and stocker calves on morbidity and mortality due to BRD using a MTC meta-analysis. These results should aid in the understanding of the effect of metaphylactic antimicrobial options on clinically important BRD outcomes.

\section{MATERIALS AND METHODS}

\section{Literature Search}

An initial literature review was conducted in April 2016 by a reviewer (KA) using, AGRICOLA (all years available), Commonwealth Agricultural Bureau (all years available), and Pubmed (all years available) for retrieval of topics relevant to the objective. The search terms included [beef OR cattle OR cow or OR bovine OR steer OR heifer OR calf OR calves] AND [metaphyl* OR prophylactic]. An initial search revealed a total of 3753 papers. Titles of peer reviewed papers published in English that included the search terms were examined for relevance. The initial search process was repeated with another independent reviewer (RL). Abstracts of relevant manuscripts were reviewed, and if agreed relevant, the full manuscript was acquired. Relevant manuscripts obtained by both reviewers were compared and only those relevant to the objective were fully reviewed. A third party reviewer was utilized if a disagreement between the first 2 reviewers occurred over the relevance of a manuscript. Studies were excluded if randomization was not reported. In addition, metaphylaxis antimicrobial had to be administered parentally to incoming feedlot or stocker calves within $48 \mathrm{~h}$ of arrival. Studies using young, lightweight veal or dairy calves were excluded; however, if age, weight, or type of cattle were specifically described and were consistent with cattle arriving at U.S. feedlots, the study was included in the analysis. Metaphylactic administration had to be the only treatment variable. Only naturally occurring BRD was used as study outcome and challenge studies were excluded from the analysis. Blinding was reported in 24 trials, and was not reported in 16 trials, all trials were included in the statistical analysis regardless of blinding criteria. In 2 trials, blinding was reported to have not been possible due to the person implementing the BRD treatment protocol having prior knowledge of previous antimicrobials administered metaphylactically (Van Donkersgoed, 2012; Van Donkersgoed and Merrill, 2013a).

\section{Data Extraction}

Outcome data comparing a metaphylactic antimicrobial to another antimicrobial or a control within each trial within each study was extracted (Larson and Step, 2012; Theurer et al., 2015). If a study contained multiple trials, the data from each trial were extracted separately. All data for each trial were extracted by a single reviewer (KA), and verified by a second reviewer (RL). A treatment arm was considered a different antimicrobial for each trial. For example, if a trial consisted of antimicrobial $\mathrm{A}$ and $\mathrm{B}$, this trial included two different treatment arms. For each trial, the following data were extracted: the interventions (antimicrobial) for each treatment arm, the number of animals enrolled in each treatment arm, and event occurrence for each treatment arm (Table 1). Event occurrence included morbidity, retreatment morbidity, and mortality related to BRD. Data were aggregated between treatment arms within a trial if the difference between those treatment arms was due to a difference in the post metaphylactic interval or route of antimicrobial administration. For example, if the difference between 2 antimicrobial groups was the dosage of the antibiotic (tilmicosin $10 \mathrm{mg} / \mathrm{kg}$ and $20 \mathrm{mg} / \mathrm{kg}$ ), then antimicrobials were aggregated to a single antimicrobial group (tilmicosin; Corbin et al., 2009). Also, if the difference between 2 
Table 1. Data extracted from 37 individual trials and 29 studies included in the mixed treatment comparison meta-analysis for each outcome event

\begin{tabular}{|c|c|c|c|c|c|c|c|c|}
\hline Treatment arms & $\begin{array}{c}\text { Number of } \\
\text { animals }\end{array}$ & $\begin{array}{l}\text { EU/ } \\
\text { group }^{1}\end{array}$ & $\begin{array}{l}\mathrm{Wt}^{2} \\
(\mathrm{~kg})\end{array}$ & $\begin{array}{l}\text { BRD morb } \\
\text { to } 60^{3}\end{array}$ & $\begin{array}{l}\text { BRD morb } \\
\text { to closeout }{ }^{4}\end{array}$ & $\begin{array}{l}\text { BRD mort } \\
\text { to closeout } 5\end{array}$ & $\begin{array}{l}\text { BRD retreat } \\
\text { to closeout }{ }^{6}\end{array}$ & Trial \\
\hline ceftiofur/gamithromycin & 1853 & $931 / 922$ & 205 & $354 / 295$ & - & - & - & (Amrine et al., 2014) \\
\hline control/gamithromycin & 87 & $44 / 43$ & 582 & $12 / 8$ & - & - & - & (Baggott et al., 2011) \\
\hline control/gamithromycin & 242 & $121 / 121$ & 390 & $32 / 8$ & - & - & - & (Baggott et al., 2011) \\
\hline control/gamithromycin & 227 & $114 / 113$ & 430 & $32 / 6$ & - & - & - & (Baggott et al., 2011) \\
\hline $\begin{array}{l}\text { tilmicosin/oxytetracycline/ } \\
\text { tulathromycin }\end{array}$ & 9910 & $\begin{array}{c}3304 / 3302 / \\
3304\end{array}$ & 300 & - & $464 / 562 / 113$ & $62 / 84 / 10$ & $179 / 218 / 26$ & (Booker et al., 2007) \\
\hline tilmicosin/ceftiofur & 11,605 & $3870 / 7735$ & 256 & - & $1116 / 2120$ & $423 / 626$ & $713 / 1222$ & (Booker et al., 2007) \\
\hline control/tilmicosin & 1000 & $200 / 800$ & 207 & - & $68 / 164$ & $27 / 54$ & $23 / 58$ & (Corbin et al., 2009) \\
\hline control/tilmicosin & 997 & $200 / 797$ & 265 & - & $137 / 374$ & $1 / 14$ & $53 / 107$ & (Corbin et al., 2009) \\
\hline control/tilmicosin & 64 & $32 / 32$ & 224 & $23 / 15$ & - & - & - & (Duff et al., 2000) \\
\hline control/oxytetracycline & 1793 & $893 / 900$ & 120 & $71 / 30$ & - & - & - & (Fazzio et al., 2015) \\
\hline control/florfenicol & 60 & $30 / 30$ & 230 & $13 / 10$ & - & - & - & (Frank et al., 2002) \\
\hline control/florfenicol & 42 & $21 / 21$ & 230 & $12 / 9$ & - & - & - & (Frank et al., 2002) \\
\hline control/tilmicosin & 57 & $28 / 29$ & 170 & $13 / 0$ & - & - & - & (Galyean et al., 1995) \\
\hline control/tilmicosin & 116 & $58 / 58$ & 191 & $19 / 7$ & - & - & - & (Galyean et al., 1995) \\
\hline control/tilmicosin & 121 & $62 / 59$ & 232 & $27 / 7$ & - & - & - & (Galyean et al., 1995) \\
\hline control/tilmicosin & 400 & $200 / 200$ & 273 & $113 / 51$ & $123 / 60$ & $0 / 2$ & $12 / 8$ & (Guthrie et al., 2004) \\
\hline control/TMS/oxytetracycline & 900 & $300 / 300 / 300$ & 325 & $139 / 133 / 97$ & $172 / 169 / 140$ & $10 / 9 / 6$ & - & (Harland et al., 1991) \\
\hline control/gamithromycin & 308 & $154 / 154$ & 293 & $64 / 34$ & - & - & - & (Lechtenberg et al., 2011) \\
\hline control/gamithromycin & 159 & $53 / 106$ & 256 & $34 / 15$ & - & - & - & (Lechtenberg et al., 2011) \\
\hline control/florfenicol & 108 & $54 / 54$ & 271 & $16 / 18$ & - & - & - & (Martin et al., 2007) \\
\hline control/tilmicosin & 199 & $100 / 99$ & 215 & $54 / 15$ & - & - & - & (McClary and Vogel, 1999) \\
\hline $\begin{array}{l}\text { control/tilmicosin/ } \\
\text { oxytetracycline }\end{array}$ & 1806 & $601 / 602 / 603$ & 300 & $254 / 117 / 157$ & - & - & - & (Morck et al., 1993) \\
\hline tilmicosin/tulathromycin & 293 & $147 / 146$ & 219 & $100 / 48$ & - & $20 / 5$ & - & (Nickell et al., 2008) \\
\hline control/gamithromycin & 250 & $125 / 125$ & 350 & $43 / 6$ & - & - & - & (Rossi et al., 2010) \\
\hline $\begin{array}{l}\text { oxytetracycline/ } \\
\text { gamithromycin }\end{array}$ & 470 & $235 / 235$ & 345 & $34 / 4$ & - & - & - & (Rossi et al., 2010) \\
\hline $\begin{array}{l}\text { tulathromycin/ } \\
\text { gamithromycin }\end{array}$ & 1136 & $568 / 568$ & 325 & $83 / 53$ & - & - & - & (Rossi et al., 2010) \\
\hline control/tilmicosin & 305 & $154 / 151$ & 337 & $35 / 8$ & - & - & - & (Schumann et al., 1990) \\
\hline control/tilmicosin & 205 & $103 / 102$ & 269 & $21 / 2$ & - & - & - & (Schumann et al., 1991) \\
\hline tilmicosin/oxytetracycline & 10,989 & $5494 / 5495$ & 281 & - & $1064 / 1239$ & $77 / 85$ & $409 / 454$ & (Schunicht et al., 2002) \\
\hline tilmicosin/ceftiofur & 385 & 194/191 & - & $14 / 18$ & - & - & - & (Step et al., 2007) \\
\hline $\begin{array}{l}\text { control/tilmicosin/ } \\
\text { tulathromycin }\end{array}$ & 2336 & $783 / 784 / 769$ & 312 & - & $112 / 45 / 16$ & $24 / 11 / 8$ & - & (Tennant et al., 2014) \\
\hline $\begin{array}{l}\text { tulathromycin/ } \\
\text { gamithromycin }\end{array}$ & 2529 & $1266 / 1263$ & 230 & $274 / 361$ & - & - & - & (Torres et al., 2013) \\
\hline tilmicosin/gamithromycin & 5000 & $2500 / 2500$ & 312 & - & $480 / 320$ & $10 / 15$ & $81 / 44$ & (Van Donkersgoed, 2012) \\
\hline tilmicosin/tildipirosin & 4500 & $2250 / 2250$ & 336 & - & $608 / 338$ & $20 / 20$ & $79 / 54$ & $\begin{array}{c}\text { (Van Donkersgoed and } \\
\text { Merrill, 2013a) }\end{array}$ \\
\hline control/tilmicosin & 4314 & $2157 / 2157$ & 348 & - & $259 / 173$ & $9 / 2$ & $53 / 33$ & $\begin{array}{l}\text { (Van Donkersgoed and } \\
\text { Merrill, 2013b) }\end{array}$ \\
\hline tilmicosin/tulathromycin & 4494 & $2250 / 2244$ & 274 & - & $315 / 67$ & $5 / 1$ & $28 / 7$ & $\begin{array}{l}\text { (Van Donkersgoed } \\
\text { et al., 2008) }\end{array}$ \\
\hline control/tilmicosin & 1096 & $550 / 546$ & 259 & $298 / 165$ & $317 / 185$ & $23 / 9$ & $50 / 22$ & (Vogel et al., 1998) \\
\hline
\end{tabular}

\footnotetext{
${ }^{1}$ Experimental units per antimicrobial group.

${ }^{2}$ Allocation weight in kilograms.

${ }^{3} \mathrm{BRD}$ morbidity cumulative incidence $\mathrm{d} 1$ to $\leq 60$ of the feeding period.

${ }^{4} \mathrm{BRD}$ morbidity cumulative incidence $\mathrm{d} 1$ to closeout of the feeding period.

${ }^{5} \mathrm{BRD}$ mortality cumulative incidence $\mathrm{d} 1$ to closeout.

${ }^{6} \mathrm{BRD}$ retreatment cumulative incidence $\mathrm{d} 1$ to closeout.
} 
treatments with the same antimicrobial was due to postmetaphylactic interval (ceftiofur 3 PMI and 7 PMI), then antimicrobials were aggregated to a single antimicrobial group (ceftiofur; Booker et al., 2006).

BRD morbidity included calves that were enrolled in the trial and had to be treated for BRD. The protocol for BRD diagnosis needed to be described in the report, and had to include rectal temperature, clinical signs consistent with BRD, and administration of an antimicrobial. If this protocol was not outlined, the corresponding author was contacted for clarification of the protocol to diagnose BRD. Two corresponding authors were contacted and responded for clarification. If the results were given as a percent of animals in each treatment arm, then the event occurrence was extracted based on the total number of animals enrolled in that trial for each of the treatment arms. If the numerator and denominator used to calculate the percent could not be distinguished, the data were excluded in the analysis.

BRD retreatment morbidity was classified as animals initially diagnosed with BRD and treated with an antimicrobial that required an additional antimicrobial for BRD. If mortality data were not provided in the trial, or BRD mortality could not be distinguished from the overall mortality events, the mortality data were excluded in the analysis.

Treatment periods were established as either $\mathrm{d} 1$ to $\leq$ 60 of the feeding period or $\mathrm{d} 1$ to the end of the feeding period, and a single event could be classified as occurring in both treatment periods. The end of the feeding period is referred to as closeout. Day 1 included the day the metaphylactic treatment was given. If the monitoring period of the study was less than $60 \mathrm{~d}$, the data were only included in the $\mathrm{d} 1$ to $\leq 60$ of the feeding period category. Trial days ranged from 7 to $60 \mathrm{~d}$ and this variability was accounted for in the analysis. If a trial included event results from d 1 to $\leq 60$ over multiple periods, the event results closest to $60 \mathrm{~d}$ was included. Any trial data that did not fall into one of these categories were excluded.

\section{Multiple Treatment Comparison Analysis}

The effectiveness of each individual treatment arm for the BRD morbidity d 1 to $\leq 60$ was examined using the binomial likelihood, complimentary log-log link, random-effects model for combining direct and indirect evidence in mixed treatment comparisons using a Bayesian approach as previously described (Higgins and Whitehead, 1996; Lu and Ades, 2004; Dias et al., 2010). This model assumes that the outcome for BRD morbidity $\mathrm{d} 1$ to $\leq 60$ is time dependent, and based on the differing lengths of each treatment arm the time until an event occurs has an exponential distribution (Dias et al., 2011). Differing days at risk were accounted for BRD morbidity d 1 to $\leq 60$, for example if a trial period was $14 \mathrm{~d}$, the days at risk would be $14 / 60=0.23 \mathrm{~d}$ at risk. Trial days were only accounted for in trials included in the BRD morbidity $\mathrm{d} 1$ to $\leq 60$ outcome. The effectiveness of each individual treatment arm for BRD morbidity, BRD mortality, and BRD retreatment $\mathrm{d} 1$ to $\leq$ closeout was examined using the binomial likelihood, logit link, randomeffects model for combining direct and indirect evidence in mixed treatment comparisons using a Bayesian approach similar to $\mathrm{d} 1$ to $\leq 60 \mathrm{~d}$ evaluation. (Higgins and Whitehead, 1996; Lu and Ades, 2004; Dias et al., 2010). This model assumes that the proportional odds assumption holds, that all trials occur within the same time period, and further days at risk would not affect the differences between events (Dias et al., 2011). The code was called through WinBUGS with $\mathrm{R}$ to fit the model with the R2Winbugs package (Dias et al., 2011).

A homogeneous variance was assumed and uniformed priors were used for the standard deviation, $\sigma$ for each of the BRD morbidity $\mathrm{d} 1$ to $\leq 60 \mathrm{~d}$, BRD morbidity $\mathrm{d} 1$ to closeout, BRD mortality $\mathrm{d} 1$ to closeout, and BRD retreatment morbidity d 1 to closeout models. Two uniform standard deviation priors were compared for each individual outcome model, $\sigma \sim$ uniform $(0,5)$ vs. $\sigma \sim$ uniform $(0,2)$, and based on narrower credibility intervals (CrIs) and lower deviance information criterion (DIC), $\sigma \sim$ uniform $(0,5)$ was used in the final code. GelmanRubin diagnostics were performed to determine best convergence for chains (Gelman and Rubin, 1992). A total of 2 chains were used for each model, each with 120,000 iterations, with the first 20,000 iterations discarded. The output from the code was the posterior mean for odds ratio between the treatment arm comparisons with corresponding 95\% CrIs. Treatment arms with the least OR and with corresponding overlapping 95\% CrIs were classified as "upper tier." Treatment arms with the greatest OR and with corresponding overlapping 95\% CrIs were classified as "lesser tier." Treatment arms in between the greatest and least OR and with corresponding overlapping 95\% CrIs were classified as "middle tier."

\section{RESULTS}

After initial screening for relevant titles and abstracts, a final list of 170 publications was retrieved and evaluated. From these publications, 29 studies, with a total of 37 trials met all inclusion criteria. The length of the trial periods ranged from 7 to $293 \mathrm{~d}$. Only BRD morbidity cumulative incidence is reported for treatment period $d$ 1 to $\leq 60$ of the feeding period and all event outcomes (BRD morbidity, mortality, and retreatment morbidity) are reported for treatment period d 1 to closeout.

Figure 1 shows a network of the different treatment arms included for each individual event outcome. BRD 
(a)

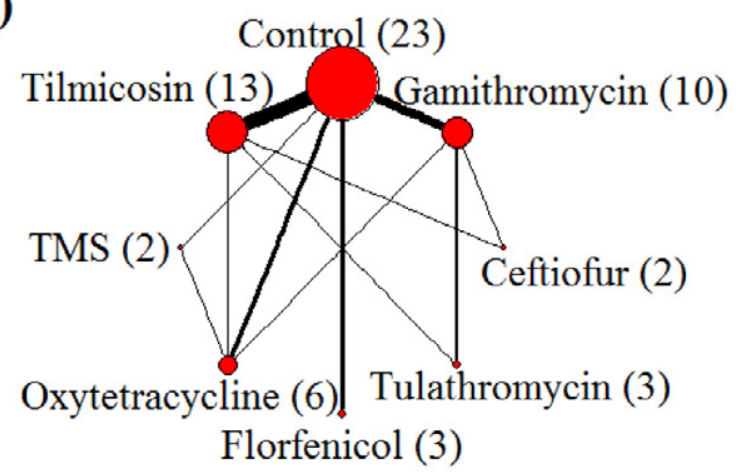

(c)

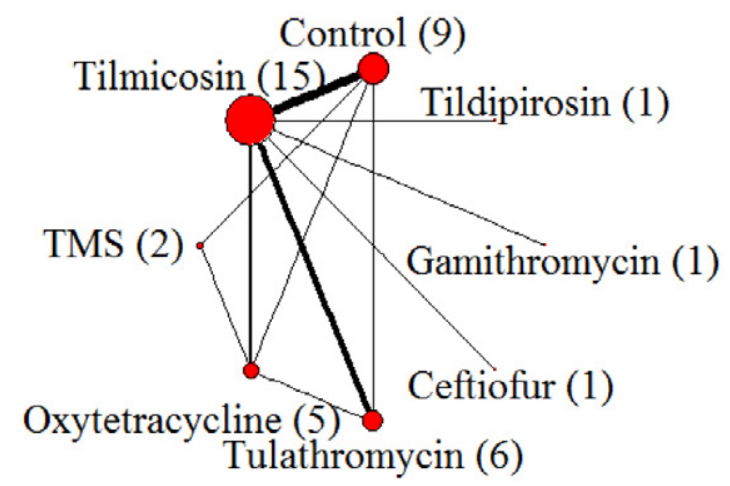

(b)

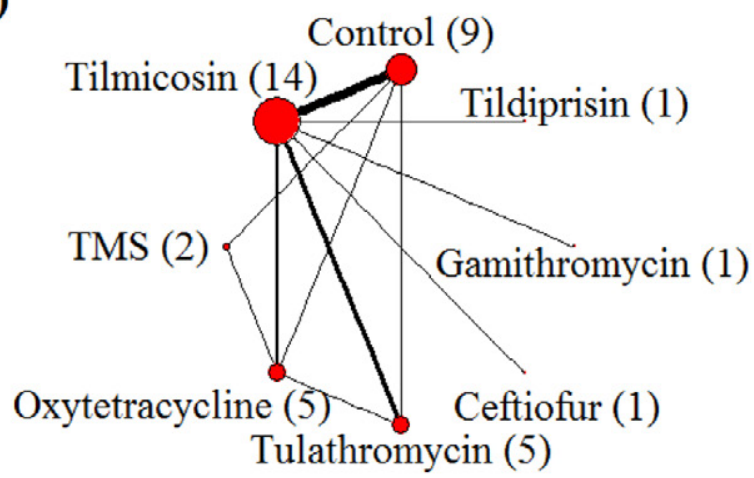

(d)

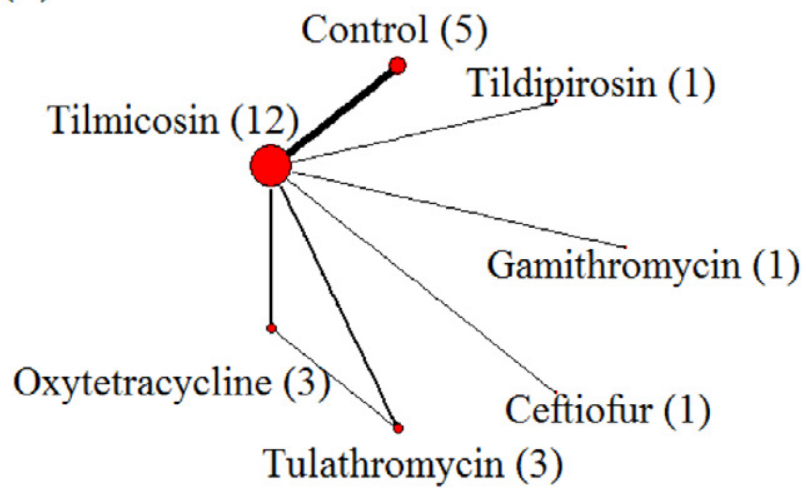

Figure 1. Network of treatment arms for the metaphylactic antimicrobial for BRD morbidity cumulative incidence $\mathrm{d} 1$ to $\leq 60$ of the feeding period (a), BRD morbidity cumulative incidence $\mathrm{d} 1$ to closeout of the feeding period (b), BRD mortality cumulative incidence $\mathrm{d} 1$ to closeout (c), and BRD retreatment morbidity cumulative incidence $\mathrm{d} 1$ to closeout (d) in the mixed treatment comparison meta-analysis. The width of the lines corresponds to the number of direct comparisons between antimicrobials, the size of the dot indicates the number of antimicrobials within each arm, and number in parenthesis corresponds to the number of comparisons for each antimicrobial.

morbidity cumulative incidence $\mathrm{d} 1$ to $\leq 60$ included 62 treatment arms from 27 trials, BRD morbidity cumulative incidence $\mathrm{d} 1$ to closeout included 37 treatment arms from 13 trials, BRD mortality cumulative incidence $\mathrm{d} 1$ to closeout included 40 treatment arms from 14 trials, and BRD retreatment morbidity cumulative incidence $\mathrm{d} 1$ to closeout included 26 treatment arms from 11 trials (Fig. 1). The maximum number of treatment arms within a trial were 3 (Harland et al., 1991; Morck et al., 1993; Booker et al., 2007; Tennant et al., 2014), all other trials contained 2 treatment arms. There were a total of 8 different metaphylactic antimicrobials (Table 1). All treatment arms were included in 4 or more trials except florfenicol, tildipirosin, and TMS. Florifenicol had 3 trials, tildipirosin had 1 trial, and TMS had 1 trial included. A placebo control was present in 25 trials.

Forest plots of the mean odds ratio (OR) comparisons between antimicrobial and control with $95 \% \mathrm{CrIs}$ for each event outcome are shown in Fig. 2. The dotted line in the center of each forest plot designates the OR equal to 1 . If the OR are equal to 1 , odds of the event occurrence are the same for the antimicrobial compared to the control; if odds are less than 1, the odds for the event occurrence are greater for the con- trol compared to the antimicrobial; if odds are greater than 1, the odds for the event occurrence are greater for the antimicrobial compared to the control. BRD morbidity cumulative incidence d 1 to $\leq 60$ "upper tier" treatment arms were tulathromycin, gamithromycin, and tilmicosin. The "middle tier" included ceftiofur and oxytetracycline, and the "lesser tier" included florfenicol and TMS. Morbidity cumulative incidence d 1 to closeout "upper tier" treatment arms included tulathromycin, the "middle tier" include tildipirosin, gamithromycin, ceftiofur, tilmicosin, and oxytetracycline, and the "lesser tier" included TMS. Mean OR for all comparisons between antimicrobials with $95 \%$ CrIs for each event outcome are shown in Table 2.

For BRD mortality cumulative incidence d 1 to closeout and $\mathrm{BRD}$ retreatment morbidity $\mathrm{d} 1$ to closeout, classifying the treatment arms into tiers was not possible due to overlapping $95 \%$ CrIs. However, there were some differences between individual antimicorbials. In Fig. 2(c), the 95\% CrIs for tulathromycin did not overlap with the $95 \%$ CrIs of tilmicosin and oxytetracycline. Overall, tulathromycin and tilmicosin has a lesser odds than the controls, and oxytetracycline is similar to the controls. The OR and 95\% CrIs of the comparison of tulathromy- 


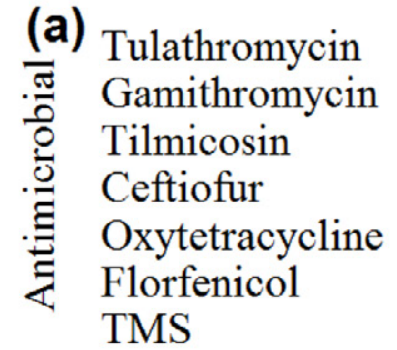

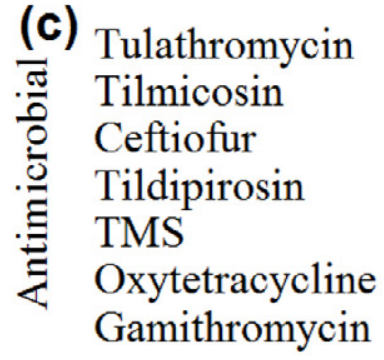

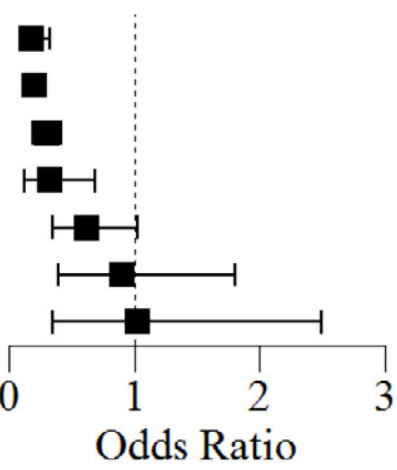

Odds Ratio
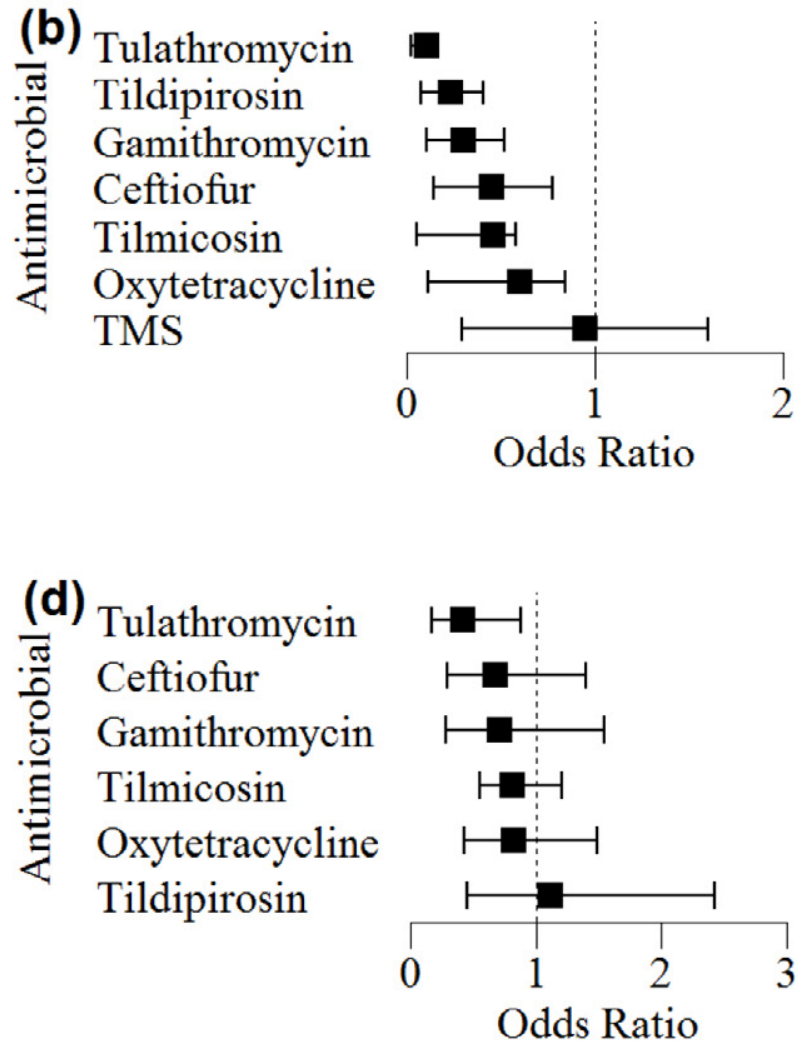

Figure 2. Forest plots of the odds ratio comparison between individual antimicrobials and control in the mixed treatment comparison with a $95 \%$ CrIs for BRD morbidity cumulative incidence $\mathrm{d} 1$ to $\leq 60$ of the feeding period (a) ${ }^{1}$, BRD morbidity cumulative incidence d 1 to close out of the feeding period $(b)^{2}$, BRD mortality cumulative incidence $\mathrm{d} 1$ to closeout $(\mathrm{c})^{2}$, and BRD retreatment morbidity cumulative incidence $\mathrm{d} 1$ to closeout $(\mathrm{d})^{2}$. ${ }^{1} \mathrm{Binomial}$ likelihood, complimentary log-log link, random-effects model. ${ }^{2}$ Binomial likelihood, logit link, random-effects model.

cin vs. tilmicosin is $0.26(0.13$ to 0.49$)$ and tulathromycin vs. oxytetracycline is 0.20 [0.08 to 0.41 ; Table $2(\mathrm{c})]$. OR for tulathromycin is different from tilmicosin and oxytetracycline, and the odds of mortality cumulative incidence $\mathrm{d} 1$ to closeout of the feeding period is 4 times greater for tilmicosin than tulathromycin, and 5 times greater for oxytetracycline than tulathromycin.

\section{DISCUSSION}

The results of the MTC meta-analysis were able to accurately identify differences between metaphylactic antimicrobials related to BRD morbidity, retreatment, and mortality. A wide variety of trials conducted between different antimicrobials were identified in the published literature. This MTC meta-analysis allows for simultaneous inference between treatment arms based on the model estimates ( $\mathrm{Lu}$ and Ades, 2004). The data included in the MTC meta-analysis performs comparisons between the direct and indirect treatments and allows precision to increase with the assumption of consistency between these antimicrobials (Salanti et al., 2008).

Veterinarians and producers establish a metaphylactic treatment protocol based on prior knowledge of the incoming group of calves risk factors, season, weight, geographic origin, prior experience, and published literature
(Ribble et al., 1995; Snowder et al., 2006; Sanderson et al., 2008; USDA, 2011). The overall goal of a metaphylactic antimicrobial is to decrease the risk and negative effect of BRD in feedlot cattle. The results from this MTC meta-analysis provide veterinarians and producers guidance to more accurately predict the expected outcomes when choosing among antimicrobials to use on incoming high-risk cattle in a feedlot or stocker operation. For example, tulathromycin has the least OR compared to all other treatment arms in BRD morbidity cumulative incidence $\mathrm{d} 1$ to $\leq 60 \mathrm{~d}$, BRD morbidity cumulative incidence d 1 to closeout, BRD mortality cumulative incidence d 1 to closeout, and BRD retreatment morbidity cumulative incidence $\mathrm{d} 1$ to closeout outcomes when compared to controls (Fig. 2). Tulathromycin is also comparable to other antimicrobials for BRD morbidity cumulative incidence $\mathrm{d} 1$ to $\leq 60$, because the $95 \%$ CrIs of tulathromycin overlaps with gamithromycin and tilmicosin (Fig. 2(a)). These 3 "upper tier" treatment arms appear comparable in the effect differences between controls for the odds of disease. Results from a MTC meta-analysis can be applied to a group of incoming cattle, if this group has a predicted BRD morbidity of $30 \%$ within the first $60 \mathrm{~d}$ of the feeding period. If all cattle are administered at arrival an "upper tier" treatment with an OR 0.1 to 0.2 , then the expected BRD morbidity would be about $4 \%$ to $8 \%$, or a 80 to $90 \%$ 
Table 2. The mean odds ratio with $95 \%$ credibility intervals for BRD morbidity cumulative incidence $\mathrm{d} 1$ to $\leq$ 60 of the feeding period (a) ${ }^{1}$, BRD morbidity cumulative incidence $\mathrm{d} 1$ to close out of the feeding period (b) ${ }^{2}$, BRD mortality cumulative incidence $\mathrm{d} 1$ to closeout (c) $)^{2}$, and BRD retreatment cumulative incidence $d 1$ to closeout $(d)^{2}$ of the mixed treatment comparison meta-analysis. The metaphylactic antimicrobial on the left for all odds ratio comparisons is the reference category

\begin{tabular}{|c|c|c|c|c|c|}
\hline $\begin{array}{l}\text { Event } \\
\text { outcome }\end{array}$ & Comparison $^{3}$ & OR & & $10 \mathrm{C}$ & \\
\hline \multirow{21}{*}{$\begin{array}{l}\text { (a) BRD } \\
\text { morbidity } \\
\text { d } 1 \text { to } \\
\leq 60\end{array}$} & tilmicosin vs. TMS & 3.59 & 1.19 & - & 9.30 \\
\hline & tilmicosin vs. oxytetracycline & 2.16 & 1.11 & - & 3.92 \\
\hline & tilmicosin vs. florfenicol & 3.15 & 1.26 & - & 6.68 \\
\hline & tilmicosin vs. tulathromycin & 0.59 & 0.27 & - & 1.14 \\
\hline & tilmicosin vs. ceftiofur & 1.10 & 0.43 & - & 2.43 \\
\hline & tilmicosin vs. gamithromycin & 0.69 & 0.39 & - & 1.15 \\
\hline & TMS vs. oxytetracycline & 0.74 & 0.24 & - & 1.74 \\
\hline & TMS vs. florfenicol & 1.11 & 0.26 & - & 3.03 \\
\hline & TMS vs. tulathromycin & 0.21 & 0.05 & - & 0.57 \\
\hline & TMS vs. ceftiofur & 0.39 & 0.09 & - & 1.14 \\
\hline & TMS vs. gamithromycin & 0.24 & 0.07 & - & 0.58 \\
\hline & oxytetracycline vs. florfenicol & 1.57 & 0.54 & - & 3.56 \\
\hline & oxytetracycline vs. tulathromycin & 0.30 & 0.11 & - & 0.63 \\
\hline & oxytetracycline vs. ceftiofur & 0.55 & 0.18 & - & 1.26 \\
\hline & oxytetracycline vs. gamithromycin & 0.34 & 0.16 & - & 0.62 \\
\hline & florfenicol vs. tulathromycin & 0.22 & 0.07 & - & 0.53 \\
\hline & florfenicol vs. ceftiofur & 0.41 & 0.11 & - & 1.12 \\
\hline & florfenicol vs. gamithromycin & 0.25 & 0.09 & - & 0.55 \\
\hline & tulathromycin vs. ceftiofur & 2.05 & 0.65 & - & 5.07 \\
\hline & tulathromycin vs. gamithromycin & 1.26 & 0.64 & - & 2.27 \\
\hline & ceftiofur vs. gamithromycin & 0.73 & 0.29 & - & 1.55 \\
\hline \multirow{21}{*}{$\begin{array}{l}\text { (b) BRD } \\
\text { morbid- } \\
\text { ity d } 1 \text { to } \\
\text { closeout }\end{array}$} & tilmicosin vs. TMS & 2.07 & 1.11 & - & 3.56 \\
\hline & tilmicosin vs. oxytetracycline & 1.29 & 0.94 & - & 1.77 \\
\hline & tilmicosin vs. tulathromycin & 0.23 & 0.16 & - & 0.32 \\
\hline & tilmicosin vs. ceftiofur & 0.97 & 0.54 & - & 1.62 \\
\hline & tilmicosin vs. gamithromycin & 0.64 & 0.35 & - & 1.08 \\
\hline & tilmicosin vs. tildipirosin & 0.50 & 0.27 & - & 0.83 \\
\hline & TMS vs. oxytetracycline & 0.67 & 0.37 & - & 1.14 \\
\hline & TMS vs. tulathromycin & 0.12 & 0.06 & - & 0.22 \\
\hline & TMS vs. ceftiofur & 0.51 & 0.21 & - & 1.04 \\
\hline & TMS vs. gamithromycin & 0.34 & 0.14 & - & 0.69 \\
\hline & TMS vs. tildipirosin & 0.26 & 0.11 & - & 0.53 \\
\hline & oxytetracycline vs. tulathromycin & 0.18 & 0.12 & - & 0.27 \\
\hline & oxytetracycline vs. ceftiofur & 0.77 & 0.39 & - & 1.36 \\
\hline & oxytetracycline vs. gamithromycin & 0.51 & 0.25 & - & 0.92 \\
\hline & oxytetracycline vs. tildipirosin & 0.39 & 0.20 & - & 0.70 \\
\hline & tulathromycin vs. ceftiofur & 4.43 & 2.17 & - & 7.88 \\
\hline & tulathromycin vs. gamithromycin & 2.94 & 1.41 & - & 5.28 \\
\hline & tulathromycin vs. tildipirosin & 2.27 & 1.10 & - & 4.06 \\
\hline & ceftiofur vs. gamithromycin & 0.71 & 0.30 & - & 1.43 \\
\hline & ceftiofur vs. tildipirosin & 0.55 & 0.23 & - & 1.12 \\
\hline & gamithromycin vs. tildipirosin & 0.84 & 0.35 & - & 1.70 \\
\hline
\end{tabular}

Continued

\section{Continued}

Event

\begin{tabular}{|c|c|c|c|c|}
\hline \multirow[t]{2}{*}{ Outcome } & \multirow{2}{*}{$\frac{\text { Comparison }^{3}}{\text { tilmicosin vs. TMS }}$} & \multirow{2}{*}{$\frac{\mathrm{OR}}{1.35}$} & \multicolumn{2}{|c|}{$95 \%$ CrIs } \\
\hline & & & 0.28 & -3.84 \\
\hline \multirow{20}{*}{$\begin{array}{l}\text { (c) BRD } \\
\text { mortal- } \\
\text { ity d } 1 \text { to } \\
\text { closeout }\end{array}$} & tilmicosin vs. oxytetracycline & 1.44 & 0.74 & -2.70 \\
\hline & tilmicosin vs. tulathromycin & 0.26 & 0.13 & -0.49 \\
\hline & tilmicosin vs. ceftiofur & 0.88 & 0.25 & -2.02 \\
\hline & tilmicosin vs. gamithromycin & 1.96 & 0.44 & -5.43 \\
\hline & tilmicosin vs. tildipirosin & 1.21 & 0.32 & -3.17 \\
\hline & TMS vs. oxytetracycline & 1.64 & 0.37 & -4.82 \\
\hline & TMS vs. tulathromycin & 0.31 & 0.06 & -1.00 \\
\hline & TMS vs. ceftiofur & 1.07 & 0.14 & -3.56 \\
\hline & TMS vs. gamithromycin & 2.86 & 0.25 & -8.95 \\
\hline & TMS vs. tildipirosin & 1.52 & 0.17 & -5.41 \\
\hline & oxytetracycline vs. tulathromycin & 0.20 & 0.08 & -0.41 \\
\hline & oxytetracycline vs. ceftiofur & 0.74 & 0.15 & -1.75 \\
\hline & oxytetracycline vs. gamithromycin & 1.52 & 0.27 & -4.60 \\
\hline & oxytetracycline vs. tildipirosin & 1.02 & 0.19 & -2.70 \\
\hline & tulathromycin vs. ceftiofur & 3.81 & 0.83 & -9.42 \\
\hline & tulathromycin vs. gamithromycin & 8.41 & 1.45 & -25.26 \\
\hline & tulathromycin vs. tildipirosin & 5.39 & 1.03 & -14.73 \\
\hline & ceftiofur vs. gamithromycin & 3.38 & 0.42 & -10.70 \\
\hline & ceftiofur vs. tildipirosin & 2.01 & 0.30 & -6.41 \\
\hline & gamithromycin vs. tildipirosin & 1.15 & 0.12 & -3.58 \\
\hline \multirow{15}{*}{$\begin{array}{l}\text { (d) BRD } \\
\text { retreat- } \\
\text { ment } \\
\text { morbid- } \\
\text { ity d } 1 \text { to } \\
\text { closeout }\end{array}$} & tilmicosin vs. oxytetracycline & 1.00 & 0.59 & -1.60 \\
\hline & tilmicosin vs. tulathromycin & 0.50 & 0.22 & -0.98 \\
\hline & tilmicosin vs. ceftiofur & 0.82 & 0.39 & -1.52 \\
\hline & tilmicosin vs. gamithromycin & 0.86 & 0.37 & -1.70 \\
\hline & tilmicosin vs. tildipirosin & 1.38 & 0.60 & 2.69 \\
\hline & oxytetracycline vs. tulathromycin & 0.52 & 0.22 & 1.01 \\
\hline & oxytetracycline vs. ceftiofur & 0.90 & 0.34 & -1.85 \\
\hline & oxytetracycline vs. gamithromycin & 0.93 & 0.33 & 2.05 \\
\hline & oxytetracycline vs. tildipirosin & 1.67 & 0.54 & 3.24 \\
\hline & tulathromycin vs. ceftiofur & 2.04 & 0.62 & -4.65 \\
\hline & tulathromycin vs. gamithromycin & 2.06 & 0.59 & -5.13 \\
\hline & tulathromycin vs. tildipirosin & 3.39 & 0.97 & -8.09 \\
\hline & ceftiofur vs. gamithromycin & 1.30 & 0.37 & -2.88 \\
\hline & ceftiofur vs. tildipirosin & 1.94 & 0.61 & -4.53 \\
\hline & gamithromycin vs. tildipirosin & 2.01 & 0.55 & -4.77 \\
\hline
\end{tabular}

${ }^{1}$ Binomial likelihood, complimentary log-log link, random-effects model.

${ }^{2}$ Binomial likelihood, logit link, random-effects model.

${ }^{3}$ The antimicrobial on the left of each comparison is the denominator in the ratio, and the antimicrobial on the right is the numerator. If the OR are equal to 1, odds of the event occurrence are the same for each antimicrobial; if odds are less than 1, the odds for the event occurrence are greater for the antimicrobial on the left; if odds are greater than 1, the odds for the event occurrence are greater for the antimicrobial on the right.

reduction in odds of being diagnosed with BRD compared to controls. Overall, this type of analysis has the potential to efficiently estimate the odds of disease which can be used to assess comparative health, performance, and economic outcomes of feedlot and stocker cattle.

Previous meta-analyses have indicated metaphylaxis can reduce $\mathrm{BRD}$ morbidity, and that reduction can be from 55\% to 29\% comparing control cattle to treated (Van Donkersgoed, 1992; Wileman et al., 2009). Mortality 
due to $\mathrm{BRD}$ has also been reported to be reduced from $3.8 \%$ to $1.8 \%$ for cattle not receiving metaphylaxis compared to those that do receive metaphylaxis (Wileman et al., 2009). The results from this MTC meta-analysis presented similar results; cattle treated with an antimicrobial have a reduced OR compared to controls for morbidity (Fig. 2) and allows producers and veterinarians to compare efficacy between antimicrobials, to determine antimicrobials that are similar (i.e., no difference), and antimicrobials that may be superior to other antimicrobials (i.e., a difference exists).

Event outcome for BRD mortality in Fig. 2(c) had overlapping $95 \%$ CrIs for all the treatment arms making full interpretation of these antimicrobial comparisons challenging. The lack of identified differences between multiple treatments arms may be due to the low incidence of mortality in feedlots (Snowder et al., 2006), and the incidence was low in the studies included in the analysis which most likely contributes to the overlapping CrIs. The lack of differences does not imply observed differences would not be higher in populations at a higher risk for BRD mortality.

In the US, $59 \%$ of all feedlot cattle are treated with a metaphylactic antimicrobial at arrival (USDA, 2011). Analyzing retreatment of cattle diagnosed with BRD after metaphylactic administration is beneficial in determining the overall affect the metaphylactic antimicrobial has on BRD morbidity, treatment success, and mortality. Retreatment for the present study refers to animals initially diagnosed with BRD and treated with an antimicrobial that required an additional antimicrobial for BRD. The results from this MTC meta-analysis for the BRD retreatment morbidity cumulative incidence were challenging to interpret, event outcome for BRD retreatment morbidity in Fig. 2(d) had overlapping 95\% CrIs for all the antimicrobial arms. An analysis to compare retreatment protocols after initial metaphylactic administration was attempted, but was unsuccessful due to few trials with similar BRD retreatment morbidity protocols that could be compared.

The prevalence of BRD morbidity differs among days following feedlot arrival (Babcock et al., 2010). The reported trial days in the treatment period $\mathrm{d} 1$ to $\leq 60$ were variable in the total days at risk for calves. Accounting for variability days at risk for treatment periods is necessary when analyzing the odds of disease for a MTC meta-analysis which we accomplished using the binomial likelihood, complimentary log-log link, random-effects model for combining direct and indirect evidence in mixed treatment comparisons using a Bayesian approach. While this model accounts for days at risk, it cannot account for a skewed distribution of morbidity case occurrence in the first $60 \mathrm{~d}$.
When a study is published, reporting all aspects of the design, such as blinding, randomization, and allocation to treatment units, is crucial to perform a MTC meta-analysis. Because of incomplete reporting, making decisions based on published literature can be hampered due to limited data and subjective prediction (Pollreisz et al., 1991; Jackson, 2006). Previous publications have reported lack of reporting of crucial aspects of trials and the influence the inclusion or exclusion of specific trials have on the final analysis of a systematic review or metaanalysis (Van Donkersgoed, 1992; O'Connor et al., 2013; Theurer et al., 2015). A limitation of this project included the limited published literature for many of the metaphylactic treatments. Inconsistency may increase when later publications are combined with earlier publications due to changes in cattle, pathogens, or management over time, and careful consideration should be made when interpreting results from a MTC meta-analysis if the data between trials appears to be inconsistent (Mills et al., 2012).

\section{Conclusion}

The results from this MTC meta-analysis identified differences between parenteral metaphylactic antimicrobial options currently available. Metaphylactic antimicrobial options appear to offer different effects on BRD morbidity and mortality odds in feedlot and stocker cattle. Further research is needed to determine the effects of different metaphylactic antimicrobials on the BRD mortality, retreatment morbidity, performance, and economics of feedlot cattle.

\section{LITERATURE CITED}

Amrine, D., B. J. White, D. Goehl, S. H. Sweiger, B. Nosky, R. K. Tessman. 2014. Comparisons of metaphylactic treatments of Zactran (gamithromycin) vs. Excede (ceftiofur crystalline free acid) in high risk, stocker calves. Int. J. Appl. Res. Vet. Med. 12:220-227.

Babcock, A. H., D. G. Renter, B. J. White, S. R. Dubnicka, and H. M. Scott. 2010. Temporal distributions of respiratory disease events within cohorts of feedlot cattle and associations with cattle health and performance indices. Prev. Vet. Med. 27:198-219. doi:10.1016/j.prevetmed.2010.09.003

Baggott, D., A. Casartelli, F. Fraisse, C. Manavella, R. Marteau, S. Rehbein, M. Wiedemann, S. Yoon. 2011. Demonstration of the metaphylactic use of gamithromycin against bacterial pathogens associated with bovine respiratory disease in a multicentre farm trial. Vet. Rec. 168:241-245. doi:10.1136/vr.c6776

Booker, C. W., S. M. Abutarbush, O. C. Schunicht, G. K. Jim, T. Perrett. 2007. Evaluation of the efficacy of tulathromycin as a metaphylactic antimicrobial in feedlot calves. Vet. Ther. 8:183-200.

Booker, C. W., O. C. Schunicht, P. T. Guichon, G. K. Jim, B. K. Wildman, T. J. Pittman, T. Perrett. 2006. An evaluation of the metaphylactic effect of ceftiofur crystalline free Acid in feedlot calves. Vet. Ther. 7:257-274. 
Camp, T. H., D. G. Stevens, R. A. Stermer, and J. P. Anthony. 1981. Transit factors affecting shrink, shipping fever, and subsequent performance of feeder calves. J. Anim. Sci. 52:1219-1224. doi:10.2527/jas1981.5261219x

Corbin, M. J., J. A. Gould, B. L. Carter, D. G. McClary, and T. A. Portillo. 2009. Effects and economic implications of metaphylactic treatment of feeder cattle with two different dosages of tilmicosin on the incidence of bovine respiratory disease (BRD)a summary of two studies. Bovine Pract.42:140-152.

DeDonder, K. D., and M. D. Apley. 2015. A review of the expected effects of antimicrobials in bovine respiratory disease treatment and control using outcomes from published randomized clinical trials with negative controls. Vet. Clin. North Am. Food Anim. Pract. 31:97-111, vi. doi:10.1016/j.cvfa.2014.11.003

Dias, S., N. J. Welton, A. J. Sutton, and A. Ades. 2011. NICE DSU technical support document 2: A generalized linear modelling framework for pairwise and network meta-analysis of randomized controlled trials, available from http://nicedsu.org.uk.

Dias, S., N. J. Welton, D. M. Caldwell, and A. E. Ades. 2010. Checking consistency in mixed treatment comparison meta-analysis. Stat. Med. 29:932-944. doi:10.1002/sim.3767

Duff, G. C., D. A. Walker, K. J. Malcolm-Callis, M. W. Wiseman, and D. M. Hallford. 2000. Effects of preshipping vs arrival medication with tilmicosin phosphate and feeding chlortetracycline on health and performance of newly received beef cattle. J. Anim. Sci. 78:267-274. doi:10.2527/2000.782267x

Fazzio, L. E., M. J. Giuliodori, W. R. Galvan, N. Streitenberger, and M. F. Landoni. 2015. A metaphylactic treatment with double dose oxytetracycline reduces the risk of bovine respiratory disease in feedlot calves. Rev. Vet. 26:89-92.

Frank, G. H., R. E. Briggs, G. C. Duff, R. W. Loan, and C. W. Purdy. 2002. Effects of vaccination prior to transit and administration of florfenicol at time of arrival in a feedlot on the health of transported calves and detection of Mannheimia haemolytica in nasal secretion. Am. J. Vet. Res. 63:251-256. doi:10.2460/ajvr.2002.63.251

Galyean, M. L., S. A. Gunter, and K. J. Malcolm-Callis. 1995. Effects of arrival medication with tilmicosin phosphate on health and performance of newly received beef cattle. J. Anim. Sci. 73:1219 1226. doi:10.2527/1995.7351219x

Gelman, A., and D. B. Rubin. 1992. Inference from iterative simulation using multiple sequences. p. 457-472. doi:10.1214/ss/1177011136

Gutherie, C. A., K. C. Rogers, R. A. Christmas, G. J. Vogel, S. B. Laudert, G. D. Mechor. 2004. Efficacy of metaphylactic tilmicosin for controlling bovine respiratory disease in high-risk northern feeder calves. Bovine Pract. 38:46-53.

Harland, R. J., G. K. Jim, P. T. Guichon, H. G. G. Townsend, and E. D. Janzen. 1991. Efficacy of parenteral antibiotics for disease prophylaxis in feedlot calves. Can. Vet. J. 32:163-168.

Higgins, J. P. T., and A. Whitehead. 1996. Borrowing strength from external trials in a meta-analysis. Stat Med 15: 2733-2749. doi:10.1002/(SICI)1097-0258(19961230)15:24<2733::AIDSIM562>3.0.CO;2-0

Ives, S. E., and J. T. Richeson. 2015. Use of antimicrobial metaphylaxis for the control of bovine respiratory disease in high-risk cattle. Vet. Clin. North Am. Food Anim. Pract. 31:341-350. doi:10.1016/j.cvfa.2015.05.008

Jackson, D. 2006. The implications of publication bias for meta-analysis' other parameter. Stat. Med. 25:2911-2921. doi:10.1002/sim.2293

Jansen, J. P., R. Fleurence, B. Devine, R. Itzler, A. Barrett, N. Hawkins, K. Lee, C. Boersma, L. Annemans, J. C. Cappelleri. 2011. Interpreting indirect treatment comparisons and network metaanalysis for health-care decision making: Report of the ISPOR task force on indirect treatment comparisons good research practices: Part 1. Value Health 14:417-428. doi:10.1016/j.jval.2011.04.002
Kelly, A. P., and E. D. Janzen. 1986. A review of morbidity and mortaltiy rates and disease occurance in north american feedlot cattle. Can. Vet. J. 27:496-500.

Larson, R. L., and D. L. Step. 2012. Evidence-based effectiveness of vaccination against Mannheimia haemolytica, Pasteurella multocida, and Histophilus somni in feedlot cattle for mitigating the incidence and effect of bovine respiratory disease complex. Vet. Clin. North Am. Food Anim. Pract. 28:97-106. doi:10.1016/j.cvfa.2011.12.005

Lechtenberg, K., C. S. Daniels, G. C. Royer, D. T. Bechtol, S. T. Chester, J. Blair, R. K. Tessman. 2011. Field efficacy study of gamithromycin for the control of bovine respiratory disease in cattle at high risk of developing the disease. Int. J. Appl. Res. Vet. Med. 9:184-192.

Lofgreen, G. P., D. G. Addis, J. R. Dunbar, and J. G. Clark. 1978. Time of processing calves subjected to marketing and shipping stress. J. Anim. Sci. 47:1324-1328. doi:10.2527/jas1978.4761324x

Lu, G., and A. E. Ades. 2004. Combination of direct and indirect evidence in mixed treatment comparisons. Stat. Med. 23:31053124. doi:10.1002/sim. 1875

Martin, G. J. V., E. L. Partida, P. N. Villalobos, C. M. Lopez, C. E. Lopez-Guerrero, A. S. Blanco. 2007. Evaluation of mass and selective metaphylaxis medication with florfenicol at feedlot entry as a tool against bovine respiratory disease under commercial conditions in Spain. Cattle Pract. 15:309-311.

McClary, D., and G. Vogel. 1999. Effect of timing of tilmicosin metaphylaxis on control of bovine respiratory disease and performance in feeder cattle. Bovine Pract. 33:155-161.

Mills E. J., J. P. A. Ioannidis, K. Thorlund, H. J. Schünemann, M. A. Puhan, G. H. Guyatt. 2012. How to use an article reporting a multiple treatment comparison meta-analysis. JAMA 308:12461253. doi:10.1001/2012.jama.11228

Mills, E. J., D. Perri, C. Cooper, J. B. Nachega, W. Ping, I. Tleyjeh, P. Phillips. 2009. Antifungal treatment for invasive candida infections: A mixed treatment comparison meta-analysis. Ann. Clin. Microbiol. Antimicrob. 8:23. doi:10.1186/1476-0711-8-23

Morck D. W., J. K. Merrill, B. E. Thorlakson, M. E. Olson, L. V. Tonkinson, J. W. Costerton. 1993. Prophylactic efficacy of tilmicosin for bovine respiratory tract disease. J. Am. Vet. Med. Assoc. 202:273-277.

Nickell, J. S., and B. J. White. 2010. Metaphylactic antimicrobial therapy for bovine respiratory disease in stocker and feedlot cattle. Vet. Clin. North Am. Food Anim. Pract. 26:285-301. doi:10.1016/j.cvfa.2010.04.006

Nickell, J. S., B. J. White, R. L. Larson, D. A. Blasi, and D. G. Renter. 2008. Comparison of short-term health and performance effects related to prophylactic administration of tulathromycin versus tilmicosin in long-hauled, highly stressed beef stocker calves. Vet. Ther. 9:147-156.

O’Connor, A. M., J. F. Coetzee, N. da Silva, and C. Wang. 2013. A mixed treatment comparison meta-analysis of antibiotic treatments for bovine respiratory disease. Prev. Vet. Med. 110:77-87. doi:10.1016/j.prevetmed.2012.11.025

O’Connor, A. M., C. Yuan, J. N. Cullen, J. F. Coetzee, N. da Silva, C. Wang. 2016. A mixed treatment meta-analysis of antibiotic treatment options for bovine respiratory disease- an update. Prev. Vet. Med. doi:10.1016/j.prevetmed.2016.07.003

Pollreisz, J. H., D. T. Bechtol, and D. W. Upson. 1991. Problems and practice in mass medication of beef cattle. Vet. Clin. North Am. Food Anim. Pract. 7:659-668. doi:10.1016/S07490720(15)31078-1 
Ribble, C. S., A. H. Meek, E. D. Janzen, P. T. Guichon, and G. K. Jim. 1995. Effect of time of year, weather, and the pattern of auction market sales on fatal fibrinous pneumonia (shipping fever) in calves in a large feedlot in Alberta (1985-1988). Can. J. Vet. Res. 59:167-172.

Roever, L., and G. Biondi-Zoccai. 2016. Network meta-analysis to synthesize evidence for decision making in cardiovascular research. Arq. Bras. Cardiol. 106:333-337. doi:10.5935/ abc. 20160052

Rossi, S. C. A., S. L. Vandoni, M. Bonfanti, and A. B. Forbes. 2010. Effects of arrival medication with gamithromycin on bovine respiratory disease in feedlot cattle in Italy. Int. J. Appl. Res. Vet. Med. 8:87-96.

Salanti, G., J. P. A. Ioannidis, J. P. T. Higgins, and J. P. A. Ades. 2008. Evaluation of networks of randomized trials. Stat. Methods Med. Res. 17:279-301. doi:10.1177/0962280207080643

Sanderson, M. W., D. A. Dargatz, and B. Wagner. 2008. Risk factors for initial respiratory disease in United States' feedlots based on producer-collected daily morbiity counts. Can. Vet. J. 49:373-378.

Schumann, F. J., E. D. Janzen, and J. J. McKinnon. 1990. Prophylactic tilmicosin medication of feedlot calves at arrival. Can. Vet. J. 31:285-288.

Schumann, F. J., E. D. Janzen, and J. J. McKinnon. 1991. Prophylactic medication of feedlot calves with tilmicosin. Vet. Rec. 128:278 280. doi:10.1136/vr.128.12.278

Schunicht, O. C., P. T. Guichon, C. W. Booker, G. K. Jim, B. K. Wildman, B. W. Hill, T. I. Ward, S. W. Bauck, J. A. Jacobsen. 2002. A comparison of prophylactic efficacy of tilmicosin and a new formulation of oxytetracycline in feedlot calves. Can. Vet. J. 43:355-362.

Shao, J. B., C. F. Ni, P. F. Duan, and Y. H. Jin. 2016. Preventive effects of different drugs on asymptomatic lower extremities deep venous thrombosis after artificial joint replacement: A mixed treatment comparison. Am. J. Ther. doi:10.1097/ MJT.0000000000000438

Smith, R. A. 1998. Impact of disease on feedlot performance: A review. J. Anim. Sci. 76:272-274. doi:10.2527/1998.761272x

Snowder, G. D., L. D. Van Vleck, L. V. Cundiff, and G. L. Bennett. 2006. Bovine respiratory disease in feedlot cattle: Environmental, genetic, and economic factors. J. Anim. Sci. 84:1999-2008.doi:10.2527/jas.2006-046

Step, D. L., T. Engelken, C. Romano, B. Holland, C. Krehbiel, J. C. Johnson, W. L. Bryson, C. M. Tucker, E. J. Robb. 2007. Evaluation of three antimicrobial regimens used as metaphylaxis in stocker calves at high risk of developing bovine respiratory disease. Vet. Ther. 8:136-147.

Tennant, T. C., S. E. Ives, L. B. Harper, D. G. Renter, and T. E. Lawrence. 2014. Comparison of tulathromycin and tilmicosin on the prevalence and severity of bovine respiratory disease in feedlot cattle in association with feedlot performance, carcass characteristics, and economic factors. J. Anim. Sci. 92. doi:10.2527/jas.2014-7814
Theurer, M. E., R. L. Larson, and B. J. White. 2015. Systematic review and meta-analysis of the effectiveness of commercially available vaccines against bovine herpesvirus, bovine viral diarrhea virus, bovine respiratory syncytial virus, and parainfluenza type 3 virus for mitigation of bovine respiratory disease complex in cattle. J. Am. Vet. Med. Assoc. 246:126-142. doi:10.2460/javma.246.1.126

Torres, S., D. U. Thomson, N. M. Bello, B. J. Nosky, and C. D. Reinhardt. 2013. Field study of the comparative efficacy of gamithromycin and tulathromycin for the control of undifferentiated bovine respiratory disease complex in beef feedlot calves at high risk of developing respiratory tract disease. Am. J. Vet. Res. 74:839-846. doi:10.2460/ajvr.74.6.839

USDA. 2011. Part IV: Health and and health management on U.S. feedlots with a capacity of 1,000 or more head. Animal and Plant Health Inspection Service, Veterinary Services, Fort Collins, CO.

Van Donkersgoed, J. 1992. Meta-analysis of field trials of antimicrobial mass medication for prophylaxis of bovine respiratory disease in feedlot cattle. Can. Vet. J. 33:786-795.

Van Donkersgoed, J. 2012. A comparison of tilmicosin to gamithromycin for on-arrival treatment of bovine respiratory disease in feeder steers. Bovine Pract. 46:46-51.

Van Donkersgoed, J., and J. K. Merrill. 2013a. Efficacy of tilmicosin and tildipirosin for on-arrival treatment of bovine respiratory disease in fall-placed feedlot calves in western Canada. Bovine Pract. 47:146-151.

Van Donkersgoed, J., and J. K. Merrill. 2013b. Efficacy of tilmicosin for on-arrival treatment of bovine respiratory disease in backgrounded winter-placed feedlot calves. Bovine Pract. 47:7-12.

Van Donkersgoed, J., J. K. Merrill, and S. Hendrick. 2008. Comparative efficacy of tilmicosin versus tulathromycin as a metaphylactic antimicrobial in feedlot calves at moderate risk for respiratory disease. Vet. Ther. 9:291-297.

Vogel, G. J., S. B. Laudert, A. Zimmermann, C. A. Guthrie, G. D. Mechor, G. M. Moore. 1998. Effects of tilmicosin on acute undifferentiated respiratory tract disease in newly arrived feedlot cattle. J. Am. Vet. Med. Assoc. 212:1919-1924.

Wellman, N. G., and A. M. O'Connor. 2007. Meta-analysis of treatment of cattle with bovine respiratory disease with tulathromycin. J. Vet. Pharmacol. Ther. 30:234-241. doi:10.1111/j.13652885.2007.00846.x

Wileman, B. W., D. U. Thomson, C. D. Reinhardt, and D. G. Renter. 2009. Analysis of modern technologies commonly used in beef cattle production: Conventional beef production versus nonconventional production using meta-analysis. J. Anim. Sci. 87:3418-3426. doi:10.2527/jas.2009-1778 